\title{
Fabrication of Microstructure Arrays on Photosensitive Glass by Femtosecond Laser
}

\author{
P.N. Wan ${ }^{1}$, C.W. Cheng ${ }^{2 *}$, H.W. Huang ${ }^{3}$, and J.S. Chen ${ }^{3,4^{*}}$ \\ ${ }^{1}$ Graduate Institute of Opto-Mechatronics, National Chung Cheng University, \\ No. 168, University Rd., Min-Hsiung, Chia-Yi 621, Taiwan \\ ${ }^{2}$ ITRI South, Industrial Technology Research Institute, \\ No. 8, Gongyan Rd., Liujia District, Tainan 734, Taiwan \\ ${ }^{3}$ Department of Mechanical Engineering, National Chung Cheng University, \\ No. 168, University Rd., Min-Hsiung, Chia-Yi 621, Taiwan \\ ${ }^{4}$ Department of Mechanical Engineering, National Chung Hsing University, \\ 250 Kuo Kuang Rd., Taichung 402, Taiwan \\ E-mail: CWCheng@itri.org.tw (C.W. Cheng) ; MichaelChen@dragon.nchu.edu.tw (J.S. Chen)
}

\begin{abstract}
A maskless technique for the fabrication of U-shaped microstructure arrays on the surface of photosensitive glass by femtosecond laser-induced modification is developed. This technique is followed by heat treatment to crystallize the modified area, and the specimen is then placed in acid solution for chemical etching. The surface roughness of the microstructures is further improved by a secondary annealing process. The fabricated photosensitive glass is used as a mold template, and replicated plano-convex cylindrical arrays by UV-replica are also presented. The focusing ability of the microlens arrays on the glass mold and replicate is demonstrated.
\end{abstract}

DOI:10.2961/jlmn.2012.01.0024

Keywords: femtosecond laser, photosensitive glass, microstructures, UV curing, microlens arrays

\section{Introduction}

Foturan glass is a type of photosensitive glass commercially available from Schott Glass Corporation [1]. Its material composition is that of lithium aluminosilicate glass doped with some cerium and silver. With its unique optical transparency in the visible wavelength, chemical stability, and thermal resistance properties, it is becoming a promising material for a wide variety of applications in microsystems, micro-fluidics, micro-optics, and bio-chips [2].

2D- or 3D-microstructures have been fabricated on the surface of or inside Foturan glass by infrared femtosecond laser pulses [2-11]. Since Foturan glass has high transmissivity $(>90 \%)$ at the infrared wavelength $[12,13]$, nonlinear multiphoton absorption only occurs at the focal point with high peak power intensity and can precisely confine the absorption regions, resulting in a more precise fabrication of the microstructures [14].

This fabrication process which uses an infrared femtosecond laser can be explained as follows. The free electrons in the conduction band are excited by multi-photon absorption, and some of the free electrons reduce the silver ions to silver atoms [15]. This is followed by heat treatment to crystallize the modified area, in which the silver atoms diffuse to form silver clusters at about $500^{\circ} \mathrm{C}$ with suitable annealing time [16]; then, the crystalline phase of the lithium metasilicate grows around the silver clusters, which act as a nucleus at about $600^{\circ} \mathrm{C}$ [3]. After the heat treatment process, the modified area is selectively etched, since the crystallization area is more soluble in HF acid solution (e.g. the etching rate is about 20:1 between the crystallization and unirradiated area [12]), and results in the formation of the fabricated microstructures.
Recently, the authors [17] prepared microlens arrays embedded in Foturan glass by femtosecond laser direct writing. The advantage of this technique was that it provided an approach for the fabrication of 3D structures with arbitrary shapes. However, the relatively low throughput of the femtosecond laser direct inducing process limits its potential for high volume manufacturing. Mass production techniques, such as embossing or injection, are suitable for the mass production of plastic parts. Recently, the authors [18] used a femtosecond laser for the surface micromachining of transparent materials, i.e. fused silica as master molds for mass production, and the replication of elastomeric PDMS as molding material was demonstrated. In the previous work [19], the replication of microchannel structures in polymers using a microstamping process with a Foturan glass stamp fabrication with $\mathrm{KrF}$ excimer laser $(248 \mathrm{~nm})$ was presented. However, the process required the use of a patterning mask and, thus, the characteristic size of the resulting structures was limited by the optical diffraction properties of the mask.

In this study we have proposed and developed a maskless technique for fabricating U-shaped microstructure arrays on the surface of Foturan glass by femtosecond laserinduced modification followed by heat treatment, chemical etching, and secondary annealing. U-shaped microstructure arrays with a width (depth) of about $100 \mu \mathrm{m}(30 \mu \mathrm{m})$ were developed. The fabricated Foturan glass was used for the master stamp, and replicated polymer microstructures, i.e. plano-convex cylindrical microlens arrays, were also developed. The replicated microstructures were then used as optical components to demonstrate their focusing ability. 


\section{Experimental}

Fig. 1 shows the schematic layout of the experimental setup for femtosecond laser machining. A regenerative amplified mode-locked Ti:Sapphire femtosecond laser (SPIT FIRE, Spectra-Physics) with a repetition rate of $1 \mathrm{kHz}$, a pulse duration of $\sim 120 \mathrm{fs}$, a central wavelength of $800 \mathrm{~nm}$, and a maximum pulse energy of $3.5 \mathrm{~mJ}$ was used. In order to adjust the energy of the laser beam, the linear polarized Gaussian beam emitted from the laser was attenuated by a rotatable half-wave $(\lambda / 2)$ plate and a polarizing beam splitter (PBS). The transmitted component of the laser beam was incident upon a beam splitter, the reflected beam was launched into a power detector, and the laser irradiation energy was measured. Meanwhile, the transmitted linearlypolarized laser beam was passed through a shutter and a series of reflective mirrors, subsequently entering a $10 \mathrm{x}$ objective lens (numerical aperture 0.26, M Plan Apo NIR, Mitutoyo). The position of the objective lens was adjusted in the vertical (i.e. Z-axis) direction, and the femtosecond laser's focused spot diameter was about $5 \mu \mathrm{m}$. Microstructures were produced by translating the sample using an $X_{-}$ $\mathrm{Y}$ stage and translating the laser-focused position using a $\mathrm{Z}$ stage.

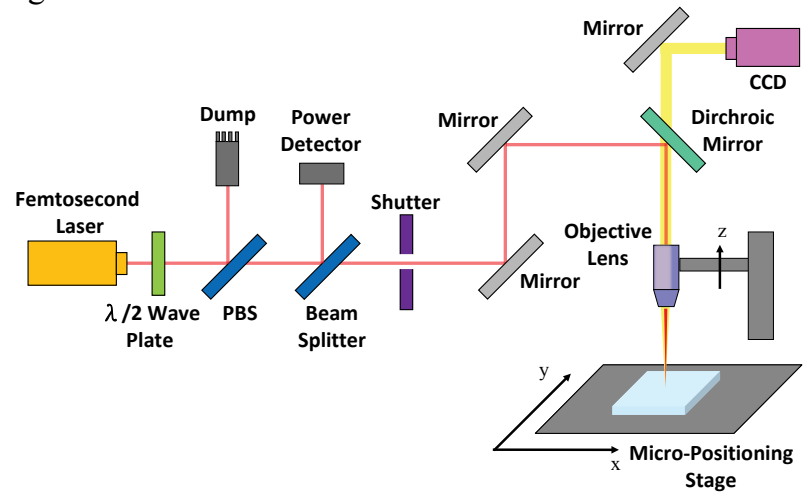

Fig. 1: Schematic layout of the experimental setup for femtosecond laser machining.

After the laser irradiation process, the Foturan glass was first heated up to $500^{\circ} \mathrm{C}$ at a rate of $5^{\circ} \mathrm{C} / \mathrm{min}$ and held at this temperature for $1 \mathrm{~h}$; the temperature was then ramped up to $600^{\circ} \mathrm{C}$ at a rate of $3^{\circ} \mathrm{C} / \mathrm{min}$ and held for another hour. Finally, the samples were cooled to room temperature under ambient conditions. After the samples cooled to room temperature, they were immersed in an $8 \%$ hydrofluoric acid (HF) etchant in an ultrasonic bath at room temperature for an etching time of $50 \mathrm{~min}$.

The UV replication experiments were realized by a UVcured imprint lithography machine with the femtosecond laser structured glass mold template. Photo-curing material (FL0881, Everwide Chemical) was used as the molding material. The processing parameters were: imprint pressure $98 \mathrm{kPa}$, imprint time $305 \mathrm{~s}$, imprint delay time $5 \mathrm{~s}$, and UV curing time $300 \mathrm{~s}$. The morphologies of the glass mold and replicate were observed by optical microscope and scanning electron microscope (SEM). Depth and roughness were measured by alpha-step and atomic force microscope (AFM), respectively.

Fig. 2 shows the schematic layout of the experimental setup for measuring the performance of the microlens arrays. An $\mathrm{He}-\mathrm{Ne}$ laser (wavelength $632.8 \mathrm{~nm}$ ) beam was incident through a spatial filter which consisted of a $10 \mathrm{x}$ lens and a pinhole. The transmitted laser beam was passed through an optical lens (focal length $100 \mathrm{~mm}$ ), resulting in a parallel laser light. The diameter of the transmitted laser beam was adjusted by an iris and, subsequently, the beam entered the microlens arrays. The laser intensity distribution at the focal plane was observed by a CCD camera.

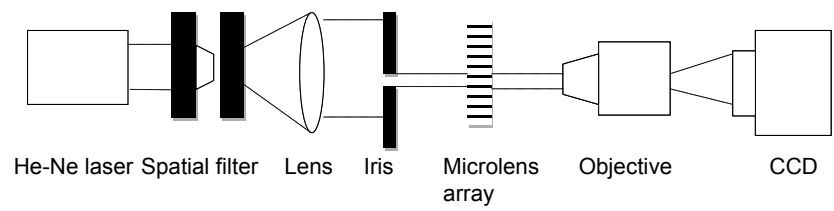

Fig. 2: Schematic layout of the experimental setup for measuring the optical performance of the microlens arrays.

\section{Results and Discussion}

Fig. 3(a) presents a microscopic image of the line pattern fabricated on the surface of the Foturan glass at a scanning speed of $0.05 \mathrm{~mm} / \mathrm{s}$ and a laser power of $0.27 \mathrm{~mW}$. Note that the specimen was unheated and unetched. It could be seen that the surface within the laser-irradiated area was slightly clearer than that within the unirradiated region. The irradiated sample was then heated, and it was found that the line pattern of the crystalline phase turned brown while the amorphous phase was still clear, as shown in Fig. 3(b). Fig. 3(c)-(d) show microscopic and SEM images of the line pattern after chemical etching, which showed that the line had a width of around $45 \mu \mathrm{m}$.

However, as shown in Fig. 3(d), some ripple-like structures were observed at the bottom of the line pattern; these will degenerate the surface quality of the replicated structures. Thus, in this study, a secondary annealing technique was used to improve the surface quality and was carried out by further annealing the sample at $560^{\circ} \mathrm{C}$ for $5 \mathrm{~h}[2,6]$. Fig. 4(a) shows the SEM image of the line pattern after the secondary annealing, and it was found that the ripple-like structures had disappeared. Fig. 4(b) shows the AFM image on the bottom of the line pattern, and the surface roughness was found to be $10.2 \mathrm{~nm}$. (a)

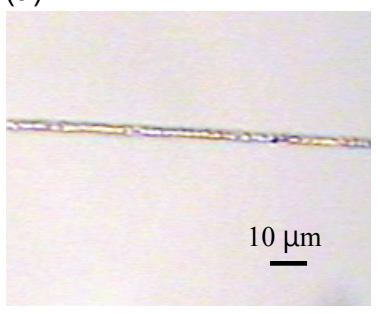

(c)

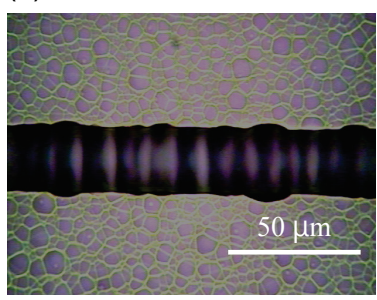

(b)

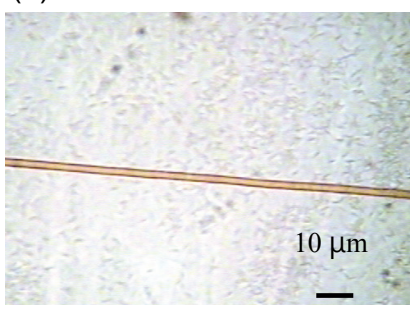

(d)

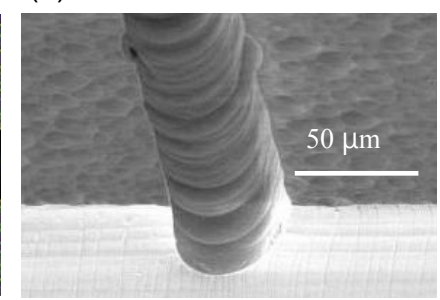

Fig. 3: Microscope images of the line pattern: (a) before heat treatment and etching, (b) after heat treatment and before etching, (c) after heat treatment and etching, and (d) SEM image of Fig. 3(c). 
(a)

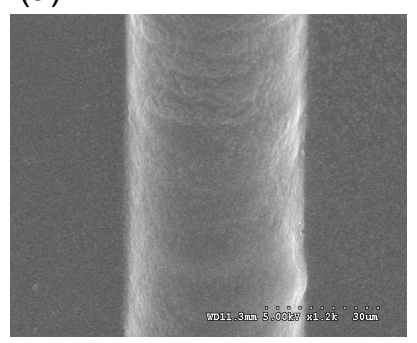

(b)

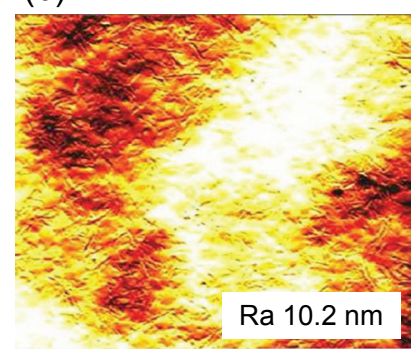

Fig. 4: The line pattern after the secondary annealing: (a) SEM image and (b) AFM image on the bottom of the line pattern.

Fig. 5 illustrates the variations in the measured fabricated line depth on the glass mold and its replication depth as a function of the laser power $(1.5 \sim 1.8 \mathrm{~mW})$ at a scanning speed of $0.05 \mathrm{~mm} / \mathrm{s}$. It was observed that the line depth increased with increasing laser power. However, the etch width was almost constant (about $100 \mu \mathrm{m}$ ), which was caused by the over-etching on the amorphous regions [20].

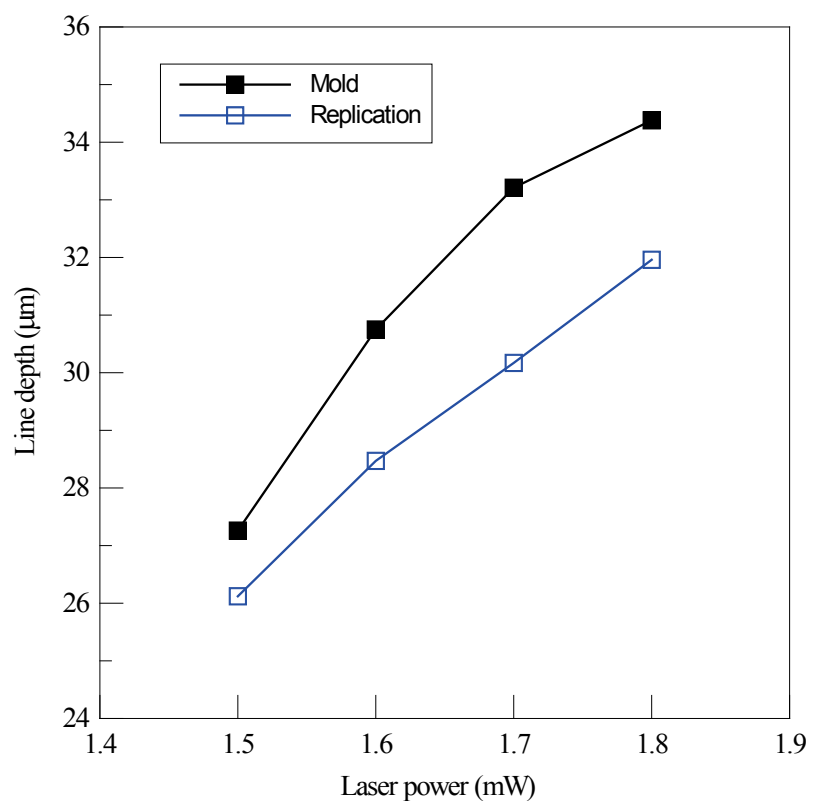

Fig. 5: Line depth on the glass mold and its replication depth as a function of laser power at a scanning speed of $0.05 \mathrm{~mm} / \mathrm{s}$.

Fig. 6 presents the fabricated microstructure line arrays on the surface of the glass mold and its replicated parts. The line width (depth) on the replicated microstructures was about $99 \mu \mathrm{m}(31.9 \mu \mathrm{m})$, and nearly equal to the line width (depth) on the mold, i.e. about $101 \mu \mathrm{m}(33.8 \mu \mathrm{m})$. The pitch of the line arrays on the glass mold and its replicate was $100 \mu \mathrm{m}$. The surface roughness on the top of the molding line pattern was $18.1 \mathrm{~nm}$. The dimensional disagreement between the mold structure and the molding structure was about $5 \%$ (calculated from the average depth difference). (a)

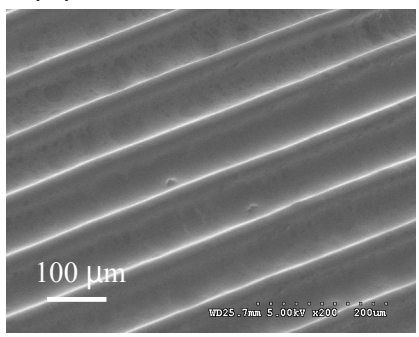

(c)

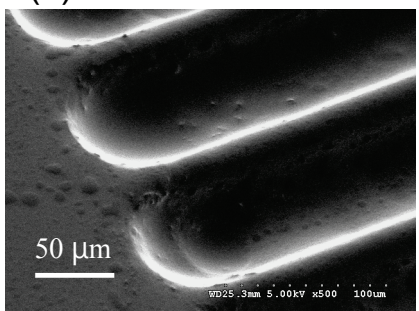

(b)

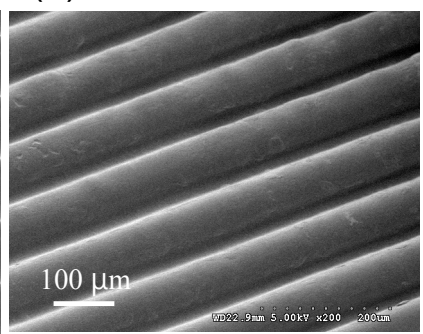

(d)

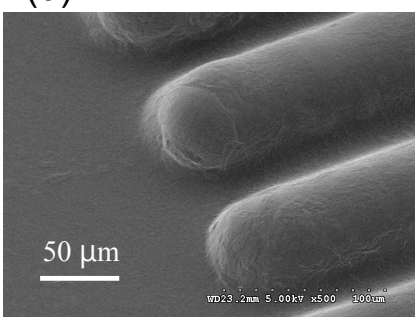

Fig. 6: SEM images of microstructure line arrays: (a) glass mold with middle part, (b) the molding of Fig. 6(a), (c) glass mold with end part, and (d) the molding of Fig. 6(c).

The focusing ability of the glass mold (plano-concave cylindrical microlens arrays) is shown in

Fig. 7. The distance between the two focus lines was about $100 \mu \mathrm{m}$, which was consistent with the planoconcave microlens arrays. The focusing ability of the replicated parts (plano-convex cylindrical microlens arrays) is shown in Fig. 8. Fig. 8(a) is the focal plane image of the line array, and the distance between the two focus lines was about $100 \mu \mathrm{m}$, which was consistent with the plano-convex microlens arrays. Fig. 8(b)-(c) show the magnification image of a focused line and its cross section profile, respectively. Therefore, we determined that the replicated microlens arrays had the potential for use as optical components.

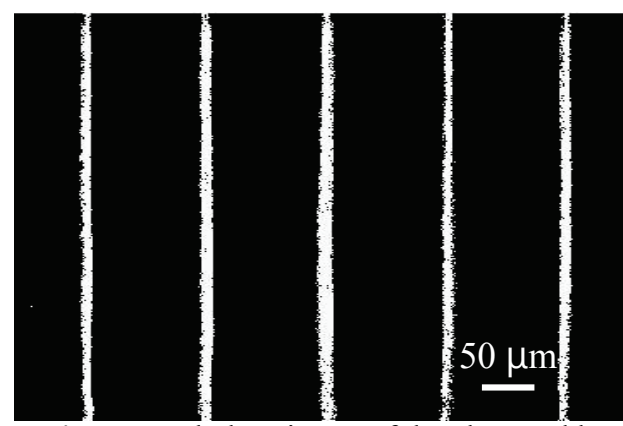

Fig. 7: Focal plane image of the glass mold.

(a)

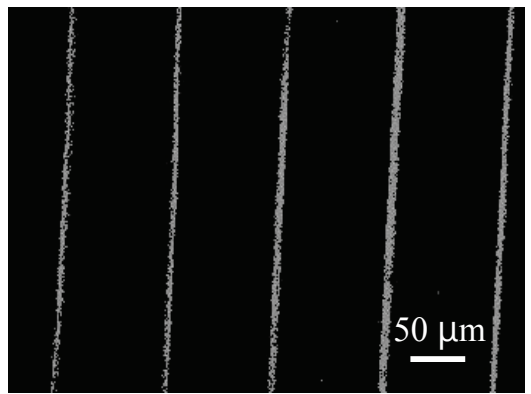


(b)

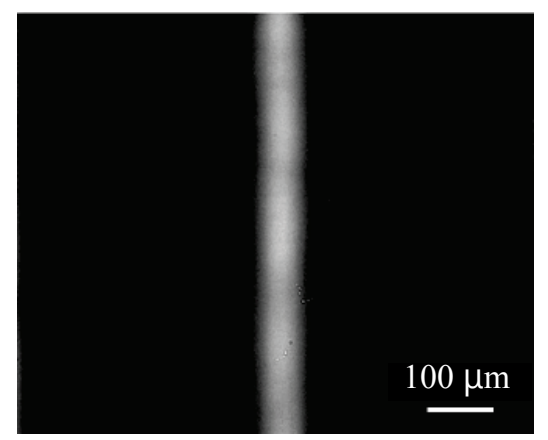

(c)

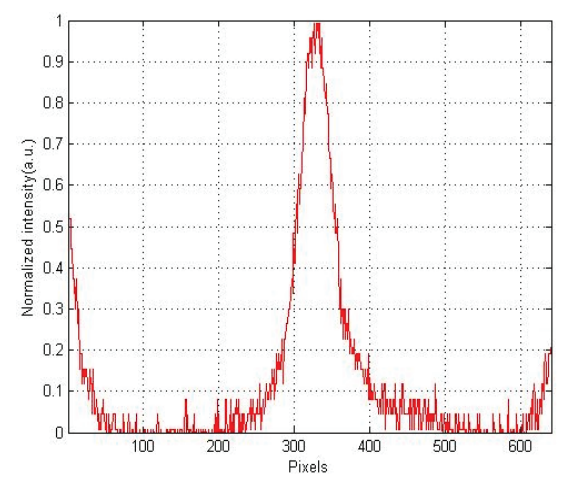

Fig. 8: (a) Focal plane image of the replicated parts, and (b) magnification image of a focused line of Fig. 8(a), and (c) cross section profile.

\section{Conclusions}

We fabricated U-shaped microstructure arrays on the surface of Foturan glass using a femtosecond laser-induced modification process followed by heat treatment, chemical etching, and secondary annealing. The experimental results showed that microstructures with a width (depth) of about $100 \mu \mathrm{m}(30 \mu \mathrm{m})$ could be fabricated on the surface of Foturan glass by carefully controlling the laser irradiation parameters. It was proven that the replicated microlens arrays from the glass mold could focus lights and, therefore, were suitable for use as optical components.

\section{Acknowledgements}

We would like to appreciate the support from the Ministry of the Economic Affairs (MOEA), Taiwan, R.O.C. for performing this project.

\section{References}

[1] T. R. Dietrich, W. Ehrfeld, M. Lacher et al., "Fabrication technologies for microsystems utilizing photoetchable glass," Micoelectron Eng., 30, (1996) 497.

[2] K. Sugioka, Y. Hanada, and K. Midorikawa: Laser PhotonicsRev., 4, (2010). 386.

[3] M. Masuda, K. Sugioka, Y. Cheng et al., “3-D microstructuring inside photosensitive glass by femtosecond laser excitation," Appl Phys A-Mater., 76, (2003). 857.

[4] K. Sugioka, Y. Cheng, and K. Midorikawa, "Threedimensional micromachining of glass using femtosecond laser for lab-on-a-chip device manufacture," Appl Phys A-Mater., 81 , (2005). 1.

[5] B. Fisette, and M. Meunier, "Three-dimensional microfabrication inside photosensitive glasses by femtosecond laser," J Laser Micro Nanoen., 1, (2006) 7.

[6] Y. Cheng, H. L. Tsai, K. Sugioka et al., "Fabrication of 3D microoptical lenses in photosensitive glass using femtosecond laser micromachining," Appl Phys A-Mater., 85, (2006). 11.

[7] Y. Z. Wu, W. Jia, C. Y. Wang et al., "Micro-hole fabricated inside FOTURAN glass using femtosecond laser writing and chemical etching," Opt Quant Electron., 39 , (2007) 1223.

[8] Z. L. Li, D. K. Y. Low, M. K. Ho et al., "Fabrication of waveguides in Foturan by femtosecond laser," J Laser Appl., 18, (2006) 320.

[9] J. M. Fernandez-Pradas, D. Serrano, S. Bosch et al., "3D features of modified photostructurable glassceramic with infrared femtosecond laser pulses," Appl Surf Sci., 257, (2011) 5219.

[10] S. Beke, L. Korosi, K. Sugioka et al., "Threedimensionally embedded indium tin oxide (ITO) films in photosensitive glass: a transparent and conductive platform for microdevices," Appl Phys A-Mater., 102, (2011) 265.

[11] F. He, H. Y. Sun, M. Huang et al., "Rapid fabrication of optical volume gratings in Foturan glass by femtosecond laser micromachining," Appl Phys AMater., 97, (2009) 853.

[12] J. Kim, H. Berberoglu, and X. F. Xu, "Fabrication of microstructures in photoetchable glass ceramics using excimer and femtosecond lasers,"JM3 Letters., 3, (2004) 478.

[13] B. Fisette, F. Busque, J. Y. Degorce et al., "Threedimensional crystallization inside photosensitive glasses by focused femtosecond laser," Appl Phys Lett., 88, (2006) 9.

[14] R. R. Gattass, and E. Mazur, "Femtosecond laser micromachining in transparent materials," Nature Photonics., 2, (2008) 219.

[15] T. Hongo, K. Sugioka, H. Niino et al., "Investigation of photoreaction mechanism of photosensitive glass by femtosecond laser," J Appl Phys., 97, (2005).

[16] F. E. Livingston, P. M. Adams, and H. Helvajian, "Examination of the laser-induced variations in the chemical etch rate of a photosensitive glass ceramic," Appl Phys A-Mater., 89, (2007) 97.

[17] C. H. Lin, L. Jiang, Y. H. Chai et al., "Fabrication of microlens arrays in photosensitive glass by femtosecond laser direct writing," Appl Phys AMater., 97, (2009) 751.

[18] F. Madani-Grasset, and Y. Bellouard, "Femtosecond laser micromachining of fused silica molds," Ops Express., 18, (2010) 21826.

[19] J. Kim, "Replication of microchannel structures in polymers using laser fabricated glass-ceramic stamp," Opt Lasers Eng., 45, (2007) 890.

[20] C. W. Cheng, J. S. Chen, P. X. Lee et al., "Fabrication of microstructures in Foturan glass using infrared femtosecond laser pulses and chemical etching," Opt Lasers Eng., 48, (2010) 811.

(Received: June 2, 2011, Accepted: November 17, 2011) 